\title{
Aspectos psicológicos determinantes do comportamento alimentar em crianças e adolescentes com sobrepeso ou obesidade
}

\author{
Determining psychological aspects of food behavior in children and adolescents with \\ overweight or obesity
}

Fernando Oliveira Pereira ${ }^{1}$

\begin{abstract}
Resumo
Sobrepeso e obesidade são condições resultantes do comportamento alimentar determinado pela ação de uma multiplicidade de fatores inerentes à funcionalidade do sujeito como sistema biopsicossocial integrado. Objetivo da investigação: Estudar aspectos psicológicos determinantes do comportamento alimentar em crianças e adolescentes com sobrepeso ou obesidade. Participantes: 50 crianças e adolescentes com diagnóstico de sobrepeso / obesidade com idades compreendidas entre 7 e 18 anos e outros 50 com caraterísticas sociodemográficas equivalentes, que constituem o grupo de controle definido pelo critério de peso e IMC dentro dos parâmetros da norma. Instrumentos metodológicos: Entrevista clínico-psicológica; questionário sociodemográfico; questionário do comportamento alimentar (QCA). Resultados: As crianças e adolescentes com sobrepeso/obesidade, comparativamente ao do grupo de controle, evidenciam IMC e expressividade de valores da dimensão "ingestão alimentar externa" mais elevados, sendo as diferenças estatisticamente significativas. Nas dimensões "restrição alimentar", menos expressiva, e "ingestão alimentar emocional", mais expressiva, as diferenças estatísticas não são significativas, quanto à quantidade de indutores envolvidos, mas são quanto à intensidade ou frequência de ação. Conclusões: Nas crianças e adolescentes com sobrepeso e obesidade o comportamento alimentar foi induzido por aspectos psicológicos de natureza sensorial e cognitivo-emocional em conjugação com provável preocupação e força de vontade deficitários em situações e circunstâncias, cuja condição exigia restrição da quantidade e tipos de alimentos ingeridos, no que concerne aos hábitos e estilo alimentar. O comportamento alimentar, na perspectiva de dependência psicológica, envolve processos de imersão emocional, colonização cognitiva, submissão motivacional e subsidiação volitiva.
\end{abstract}

Palavras-chave: comportamento alimentar; sobrepeso; obesidade infantil.

\begin{abstract}
Overweight and obesity are conditions resulting from eating behaviors determined by the action of a multiplicity of factors inherent to the subject's functionality as an integrated biopsycho-social system. Research objective: Studying psychological aspects that determine eating behavior in overweight or obese children and adolescents. Participants: 50 children and adolescents diagnosed with overweight/obesity, aged between 7 and 18 years, and another 50 with equivalent sociodemographic characteristics, which constitute the control group defined by weight and BMI criteria within standardized parameters. Methodological instruments: Clinical-psychological interview; sociodemographic questionnaire; Eating Behavior Questionnaire (EBQ). Results: Children and adolescents with overweight/obesity, compared to those in the control group, show significantly higher BMI and values of the "external food intake" dimension, with statistically significant differences. In the dimensions "food restriction" (less expressive) and "emotional food intake" (more expressive), the
\end{abstract}

\footnotetext{
${ }^{1}$ Universidade Lusófona de Lisboa. E-mail: fopereira@sapo.pt
} 
statistical differences are not significant, as to the number of inducers involved, but relate to the intensity or frequency of action. Conclusions: In overweight and obese children and adolescents, eating behavior was induced by psychological aspects of a sensory and cognitiveemotional nature in conjunction with probable concern and deficient willpower in situations and circumstances, whose condition required restriction of the amount and types of food eaten, concerning eating habits and style. Eating behavior, in the perspective of psychological dependence, involves processes of emotional immersion, cognitive colonization, motivational submission, and volitional subsidization.

Keywords: eating behavior; overweight; child obesity.

\section{Introdução}

O sujeito portador de sobrepeso ou obesidade está inserido em um sistema biopsicossocial, integrado de múltiplos fatores provenientes de naturezas diversas, cuja complexidade estrutural e funcional opera em vários níveis e dimensões, constituindo-se de aspectos e componentes ${ }^{1,2,3,4}$.

As causas do sobrepeso e da obesidade têm origem numa panóplia de fatores como a genética, fatores ambientais, condições sociais, pressões econômicas, estilos de vida familiares ou de outros problemas de saúde. Contudo, existem outros fatores que potenciam o excesso de peso, sendo um dos mais relevantes os comportamentos alimentares pouco saudáveis. Os hábitos alimentares e o sedentarismo são os principais responsáveis pelo aumento da prevalência da obesidade infantil; por isso, a prevenção dever-se-á incidir na promoção da alimentação saudável e na atividade física ${ }^{5,6}$.

Em Portugal, no espaço de tempo de uma década, segundo os dados da $5^{\mathrm{a}}$ fase do COSI Portugal (Sistema de Vigilância Nutricional Infantil do Ministério da Saúde) houve diminuição do excesso de peso nas crianças, sendo que em 2008 eram 37,9\% e em 2019 eram 29,6\%. Entretanto, o COSI (Childhood Obesity Surveillance Initiative), embora confirme a diminuição da prevalência de excesso de peso e obesidade infantil em Portugal, não elimina a necessidade de manter a preocupação de vigilância dos hábitos alimentares no país; porque regista-se que $40 \%$ dos adolescentes bebem refrigerantes diariamente, metade consome produtos hortofrutícolas abaixo do recomendável e mais de $20 \%$ consomem açúcar acima dos níveis recomendados. Os dados da mesma organização revelam que a prevalência da obesidade infantil aumentou com a idade: $15,3 \%$ das crianças de 8 anos são obesas, incluindo $5,4 \%$ com obesidade severa; valor que é de $10,8 \%$ em crianças de 6 anos $(2,7 \% \text { obesidade severa })^{7}$.

Atualmente, existe unanimidade que a obesidade é uma doença crónica com etiologia multifatorial, envolvendo aspectos genéticos, psicológicos, sociais, culturais e ambientais, cujas comorbilidades afetam a qualidade de vida e implicam riscos para a saúde dos pacientes; por isso, a abordagem terapêutica também deve assentar numa perspetiva multidisciplinar. Na obesidade, a relação entre os vários elementos que a configuram é dinâmica e simultânea; daí que abordagens terapêuticas fragmentadas correm o risco de serem pouco eficazes ${ }^{8,9}$.

O comportamento alimentar que conduz ao sobrepeso e à obesidade decorre da influência midiática que promove o consumo de alimentos inadequados, por um lado, e, por outro, um padrão ideal de magreza; a economia liberal assente no marketing e na publicidade que estimula o consumismo; os interesses da indústria da alimentação não saudável; a complexidade das relações familiares envolvidas, a discriminação social, as dificuldades subjetivas de cada um, entre outros ${ }^{10,11,12}$.

A obesidade infantil e adulta tem aumentado tão significativamente que alguns autores a consideram uma epidemia e um problema de saúde pública ${ }^{10,12,13,14,15}$. Além de ser um problema de saúde pública, também é uma questão social, devido ao estigma atribuído às pessoas com excesso de peso $^{16}$. Em 5\% dos casos de obesidade 
infanto-juvenil a etiologia é endógena, decorrendo de fatores genéticos $\mathrm{e}$ neuroendócrinos, mas em $95 \%$ dos casos a origem é exógena, sendo determinada por fatores externos de ordem variada: desmame afetivo precoce, rotinas sedentárias e actividade física escassa, alimentação nutricional hipercalórica e dinâmicas familiares disfuncionais ${ }^{17}$. Estima-se que $25 \%$ das crianças e $80 \%$ dos adolescentes com sobrepeso ou obesidade mantêm-se obesos na idade adulta ${ }^{18}$. A obesidade infanto-juvenil acarreta complicações de natureza psicossocial na vida dos indivíduos, podendo afetar a autoestima, em função da estigmatização, da dificuldade de aceitação da autoimagem corporal, do sentimento de fracasso, de inferioridade e da vivência de situações de bullying $^{19}$. O impacto da obesidade na saúde mental reporta-se a alterações emocionais de ansiedade, depressão, irritabilidade, medo, raiva, tristeza, autoestima, imagem corporal negativa e sofrimento psíquico associado ao bullying. Níveis significativos de stresse, relacionado com a sintomatologia ansiogénica, timidez, medos excessivos e rejeição social, provocam baixa autoestima ${ }^{20,21}$, autoconceito negativo decorrente da perceção negativa de si e da estigmatização física, advindas de brincadeiras depreciativas ${ }^{22}$.

A obesidade na adolescência está frequentemente associada à insatisfação com a imagem corporal e ao bullying ${ }^{23,24}$. A percepção e atitude das crianças que reconhecem ter excesso de peso, principalmente quando é devido a serem alvo de bullying na escola e em casa, interfere na sua vida quotidiana, no desenvolvimento de atividades físicas, nas relações sociais e na saúde mental ${ }^{25}$.

As questões de saúde mental na obesidade são predominantemente o stresse, a ansiedade e a depressão, os quais quase sempre resultam da insatisfação corporal, percepção da autoimagem negativa, baixa autoestima e associação pejorativa face aos padrões de estética corporal, apresentando-se muitas vezes como fatores de risco a desencadear reacções ansiosas e depressivas ${ }^{26}{ }_{\text {al }}$ Também em crianças e adolescentes com sobrepeso os sintomas depressivos e de ansiedade são os mais prevalecentes, mas outros aspectos de natureza psicológica e psicossocial como o temperamento, o stresse como fator responsável pelo aumento do nível de colesterol, coping maladaptativo e contextos de risco psicossocial são igualmente fatores que exercem influência negativa.Na prevenção do sobrepeso e da obesidade infantil existem sugestões de autores para dispensar mais atenção às questões relacionadas com a saúde materna, considerando que esta é um fator influente no desenvolvimento da criança e, consequentemente, também nas questões relacionadas com a obesidade infantil $^{27}$.

O sedentarismo é um comportamento habitualmente recorrente nos adolescentes, sendo fator de risco para a obesidade e contribuindo para a reduzida queima de calorias e acumulação de gordura corporal devido às rotinas diárias que passam por déficit de actividades físicas e domínio de atividades como ver televisão e videogames. Além disso, a falta de apoio familiar e o baixo suporte afetivo e emocional interferem negativamente no tratamento e enfrentamento psicossocial da obesidade; a dificuldade dos pais em negar alimentos em excesso aos filhos na hora das refeições e em implementar medidas que conduzam à alimentação mais saudável, contribui para o aumento de peso e o risco de comorbilidades ${ }^{28,29}$

A literatura revela a dificuldade dos familiares compreenderem e entenderem a obesidade nos filhos adolescentes, por eles mesmos, pais e avós, padecerem de quadro semelhante, tornando-se prejudicial na adoção de estratégias adequadas para enfrentar o problema; daí ser recomendável implementar medidas de educação para saúde com intuito de alcançar mudanças dos padrões familiares ${ }^{20,21,29}$.

No plano da intervenção, em vez de estratégias de controle e prevenção, através 
de programas prescritivos, verticais e culpabilizantes, propõem-se ações de prevenção baseadas na lógica de responsabilidade partilhada ${ }^{16}$.

Um aspecto possível de influenciar a ineficiência dos tratamentos na obesidade infantil é a percepção distorcida dos pais sobre o excesso de peso dos seus filhos ${ }^{30}$. As intervenções que não envolvem os pais são menos eficazes ${ }^{14,31}$. É importante envolver o conjunto familiar, escutar os filhos e os pais e compreender as relações estabelecidas no sistema familiar, porque o comportamento alimentar é aprendido. As narrativas das crianças não são muito diferentes das dos pais. Há tendência para a obesidade se repetir entre gerações, sendo fatores determinantes neste processo o ambiente familiar $\mathrm{e}$ os hábitos alimentares da família $^{32}$. A análise de conteúdo das narrativas das crianças com excesso de peso e dos seus pais revela cinco categorias temáticas: significado da comida, percepção social das pessoas com sobrepeso, influência do excesso de peso infantil no quotidiano, a relação com profissionais da saúde e os motivos para a não adesão ao tratamento ${ }^{11}$.

Para compreender a obesidade infantil e pensar intervenções mais eficazes deve-se considerar o sentido atribuído à obesidade, às relações familiares, às condições socioeconómicas e todos os elementos que circundam o fenômeno. Portanto, deve-se focar na pessoa e não na obesidade $^{11}$.

A obesidade infantil assume contornos de cronicidade, decorrendo do acúmulo excessivo de massa adiposa desde o nascimento até o final da infância, acarretando prejuízos para a saúde; visto que o excesso de peso, embora provoque distúrbios que não são fatais, comprometem significativamente a qualidade de vida futura. Além disso, o excesso de peso na idade escolar tende a estender-se ao longo do processo de desenvolvimento, tendo consequências nefastas para a saúde na idade adulta ${ }^{33}$.
O sucesso na abordagem do sobrepeso/obesidade, quando da implementação de novas estratégias de intervenção, depende particularmente dos conhecimentos detidos acerca dos determinantes comportamentais e psicossociais dos sujeitos a quem se dirige $\mathrm{a}$ ação ${ }^{34}$.

As atitudes e comportamentos alimentares das pessoas são resultado do processo de socialização e desenvolvimento; por isso, tradicionalmente são aprendidas no seio da família, sofrem influência dos pares, das experiências de cidadania, dos conhecimentos obtidos na escola e informação veiculada pelos meios de comunicação social ${ }^{35}$. Naturalmente, o ato de comer é um processo biológico, mas é a cultura que determina a forma como os seres humanos se comportam e fazem as suas escolhas alimentares ${ }^{36,37}$. Portanto, o que se come e a quantidade de alimentos ingeridos são, em grande parte, influenciados pelo património cultural, atitudes e práticas familiares ${ }^{38}$; contudo existem alterações do comportamento alimentar na infância que decorrem de dificuldades em se integrarem no meio social, apresentando manifestações do foro psicológico como ansiedade, depressão, baixa autoestima, distorção da percepção da imagem corporal e outros $39,40,41$.

Além disso, a sociedade ao instituir padrões de imagem corporal ideal faz com que surjam estigmas de se ser "gordo", sentindo-se as crianças inferiorizadas face a outras; daí a obesidade poder ser vista como condição para a estigmatização, exclusão e discriminação social ${ }^{42,43,44,45}$.

As crianças e adolescentes com ou em risco de terem distúrbios alimentares revelam atitudes inadequadas à ingestão e restrição alimentar; por exemplo, episódios de ingestão compulsiva e comportamentos compensatórios devido à incapacidade para controlar e gerir adequadamente o processo de ingestão alimentar ${ }^{43,46,47}$.

Os determinantes do comportamento alimentar não assentam 
exclusivamente em mecanismos endógenos de natureza biológica, sendo importante a compreensão da experiência subjetiva do apetite $\mathrm{e}$ as preferências por alimentos específicos. A regulação do apetite está associada ao estado de saciedade do sujeito, havendo participação tanto de mecanismos endógenos de natureza biológica como de natureza psicológica e também de mecanismos exógenos de natureza ambiental $^{48}$. Existe estreita relação entre as categorias de apetite e saciedade, a qual é visível na análise da seleção de alimentos e bebidas, motivação para ingerir, preferências e desejos individuais, consumo calórico, envolvendo as sensações de fome e saciedade, assim como diversos fatores de ordem cognitiva e emocional no processo de regulação do apetite; assim evidencia o esquema e a descrição da cascata da saciedade, resultando em parte significativa do padrão recorrente do comportamento $\operatorname{alimentar}^{48,49}$.

As estratégias de prevenção e de intervenção no âmbito da obesidade infantil não se deve limitar à mudança de hábitos alimentares, devendo ser um processo dinâmico de construção de um novo estilo de vida, levando sempre em linha de conta aspectos que envolvem questões relacionadas com a saúde, o bem-estar físico, psicológico e social dos sujeitos.
A compreensão
do desenvolvimento do apetite na infância pode ser uma questão importante no que concerne ao esclarecimento dos caminhos que conduzem ao ganho de peso ao longo da vida. ${ }^{4,48,50}$.

A existência de uma multiplicidade de fenômenos, relacionados com o comportamento alimentar e reportados a diversos níveis de funcionamento do sujeito da ação, remete para a tentativa de sistematização de caraterísticas, aspectos e dimensões envolvidas na problemática do sobrepeso e da obesidade. Estritamente ao nível da funcionalidade psicológica, e tomando por referenciais de orientação a concepção teórica sistémico-integrativa do psiquismo humano ${ }^{4}$, entende-se que o comportamento alimentar no contexto de sobrepeso e obesidade pode ser abordado no plano de mudanças operadas no seio das dimensões cognitiva, emocional, motivacional e volitiva, cujas alterações configuram o desenvolvimento de uma tipologia de dependência, em que estarão envolvidos processos de imersão emocional, colonização cognitiva, submissão motivacional e subsidiação volitiva.

Por conseguinte, o objetivo da investigação consiste em estudar aspectos psicológicos determinantes do comportamento alimentar, responsáveis pelo sobrepeso e obesidade em crianças e adolescentes.

\section{Materiais e Métodos}

\section{Amostra e tipo de estudo}

Amostra constituída

por participantes que foram referenciados por médicos de medicina geral e familiar ou por pediatras à consulta de pediatria do âmbito da obesidade por terem excesso de peso, o qual em parte dos sujeitos se enquadra na obesidade infantil; por isso, passaram a ter acompanhamento médico em clínicas situadas na Região de Saúde de Lisboa e Vale do Tejo, com a finalidade de controlar os fatores que eventualmente pudessem estar na origem do problema.

Os participantes do grupo de controle foram selecionados aleatoriamente desde que cumprissem os seguintes critérios: Não ter excesso de peso; ter idade, escolaridade e outras caraterísticas sociodemográficas idênticas às do grupo experimental.

As questões éticas foram salvaguardadas recolhendo consentimento informado e esclarecido referente a cada participante individualmente junto do próprio, dos seus progenitores e do médico que o seguia na consulta; no caso do grupo de controle o consentimento envolveu o participante e os seus progenitores. A investigação foi realizada no âmbito de instituições privadas e não públicas, como 
são os consultórios e clínicas privadas que prestam serviço à comunidade; por isso, além do assentimento livre e esclarecido dado pelos diversos intervenientes acima reportados, também teve a anuência e permissão das respectivas entidades, cujas direções administrativas e clínicas analisaram o processo e deram parecer ético favorável, em consonância com o que habitualmente é deliberado nas comissões de ética.

\section{Participantes}

Tabela 1. Caracterização sociodemográfica e condição clínica dos participantes

\begin{tabular}{|c|c|c|c|}
\hline \multicolumn{2}{|c|}{ Características } & $\begin{array}{c}\text { Diagnóstico de } \\
\text { excesso de peso / } \\
\text { obesidade }\end{array}$ & Grupo de controle \\
\hline \multirow{3}{*}{ 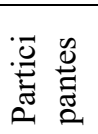 } & Total & 50 & 50 \\
\hline & Sexo masculino & $19(38 \%)$ & $18(36 \%)$ \\
\hline & Sexo feminino & $31(62 \%)$ & $32(64 \%)$ \\
\hline \multicolumn{2}{|c|}{ Intervalo etário } & $7-18$ anos & $8-18$ anos \\
\hline \multirow{3}{*}{. } & $7-10$ anos & $25(50 \%)$ & $12(24 \%)$ \\
\hline & $10-14$ anos & $20(40 \%)$ & $20(40 \%)$ \\
\hline & $14-18$ anos & $5(10 \%)$ & $18(36 \%)$ \\
\hline \multicolumn{2}{|c|}{ Estado Civil: solteiro } & $50(100 \%)$ & $50(100 \%)$ \\
\hline \multirow{4}{*}{ 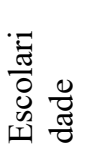 } & $1^{\circ}$ ciclo: $2^{\circ}, 3^{\circ}$ e $4^{\circ}$ ano & $21(42 \%)$ & $12(24 \%)$ \\
\hline & $2^{\circ}$ ciclo: $5^{\circ}$ e $6^{\circ}$ ano & $10(20 \%)$ & $14(28 \%)$ \\
\hline & $3^{\circ}$ ciclo: $7^{\circ}, 8^{\circ}$ e $9^{\circ}$ ano & $18(36 \%)$ & $16(32 \%)$ \\
\hline & Secundário: $10^{\circ}, 11^{\circ}$ e $12^{\circ}$ ano & $1(2 \%)$ & $8(16 \%)$ \\
\hline \multirow{6}{*}{ 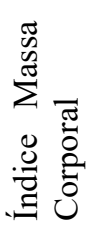 } & Intervalo IMC & $22,60-40,34$ & $18,5-26,30$ \\
\hline & IMC $18-20$ & $0(0 \%)$ & $34(68 \%)$ \\
\hline & IMC $20-25$ & $2(4 \%)$ & $12(24 \%)$ \\
\hline & IMC $25-30$ & $32(64 \%)$ & $4(8 \%)$ \\
\hline & IMC $30-35$ & $12(24 \%)$ & $0(0 \%)$ \\
\hline & IMC $35-40$ & $4(8 \%)$ & $0(0 \%)$ \\
\hline
\end{tabular}

\section{Caracterização e tipo de estudo}

A investigação consistiu num estudo quantitativo de tipologia quasiexperimental com a implementação de abordagem comparativa entre dois grupos: o experimental e o de controle.

O objetivo da investigação consiste em estudar caraterísticas psicológicas determinantes do comportamento alimentar em crianças e adolescentes com sobrepeso/obesidade, tendo motivado a colocação da seguinte hipótese de trabalho - o comportamento alimentar de crianças e adolescentes com sobrepeso ou obesidade é determinado por fatores de natureza psicológica de ordem sensorial e cognitivoemocional.

\section{Delineamento da pesquisa}

No período de tempo entre março e junho de 2020 foram identificados e referenciados os participantes da pesquisa. Depois disso, no mesmo período, procedeuse ao contato com os participantes e respectivos progenitores para obtenção do consentimento informado e esclarecido. Os dados foram coletados no período que medeia junho e novembro de 2020, em horários variados de acordo com a disponibilidade das clínicas, dos participantes e dos seus progenitores. Os instrumentos metodológicos foram aplicados individualmente e na presença do investigador, $o$ qual se manteve em permanente interação comunicativa com o participante desde o início até ao final da sessão, esclarecendo as instruções, questões em que o participante evidenciava dúvidas de compreensão e nas escalas de resposta em termos de objetivação de valores. 


\section{Critérios de Inclusão e Exclusão}

Os critérios de inclusão que orientam a seleção dos participantes na pesquisa consistem em ter diagnóstico de sobrepeso ou obesidade, IMC correspondente ao diagnóstico, não padecer de outras patologias, em particular do foro psiquiátrico, grupo experimental, e ausência de sobrepeso ou obesidade e de outras patologias, IMC dentro dos parâmetros da norma, no grupo de controle.

\section{Procedimentos}

Depois de terem sido explicadas, ao participante, as etapas do processo de avaliação dá-se início à sessão. Em primeiro lugar foi aplicado o questionário sociodemográfico, procedendo-se à recolha dos dados sociodemográficos. A seguir foi aplicado o questionário do comportamento alimentar, instruindo o participante a dar uma resposta qualitativa de "sim" ou "não" existir a atitude ou comportamento, em si, reportado na afirmação correspondente. Em seguida pede-se ao participante que atribua a frequência ou intensidade com que os respectivos comportamentos estão presentes. Por fim, é encetada uma entrevista semiestruturada com 0 participante, orientada para a obtenção de informações de natureza clínica, psicológica e psicossocial acerca de si com a finalidade de ajudar a compreender melhor algo que não tivesse ficado claro nas respostas aos questionários.

\section{Instrumentos metodológicos Cálculo do Índice de Massa Corporal (IMC)}

$\mathrm{Na}$ infância o status nutricional é avaliado com base na composição corporal e nos parâmetros relativos ao crescimento, sendo a desnutrição definida como déficit e a obesidade como excesso de gordura corporal. Existem vários métodos ou instrumentos para medir a massa corporal, mas a antropometria é o único método universal aplicável na prática clínica, porque não é dispendioso, nem invasivo, disponível para ter acesso ao peso e à composição do corpo humano, refletindo fatores tão importantes como a saúde, a nutrição, o risco e a sobrevivência ${ }^{51}$. Nesse sentido, um dos indicadores mais razoáveis é o IMC (Índice de Massa Corporal), o qual tem sido associado ao aumento de pressão arterial, ao perfil de susceptibilidade à lipoproteína, diabetes não dependentes de insulina e lesões de arterosclerose ${ }^{52}$. Em estudos sobre o crescimento verificou-se que jovens com sobrepeso registaram aumento de risco de morbilidade associado à obesidade comparativamente a adolescentes magros ${ }^{53}$. Num outro estudo que acompanhou crianças do nascimento aos 21 anos de idade, demonstrou-se que o RA (Recuperação da Adiposidade) precoce se encontra significativamente associado a um maior IMC e à dobra cutânea subescapular na idade de $21 \operatorname{anos}^{54}$.

Assim, com base nas medidas de peso em quilogramas e estatura em metros, efetuadas em todos os participantes, foi calculado o Índice de Massa Corporal (IMC) através da aplicação da fórmula de Quetelet: $\mathrm{IMC}=\mathrm{Kg} / \mathrm{m}^{2}{ }^{55}$

\section{Entrevista clínico-psicológica}

Entrevista de tipologia clínica no formato semiestruturado orientada aos aspectos psicológicos relacionados com a funcionalidade do sujeito nas diversas dimensões da sua vida. A utilização da entrevista nesse formato tem a finalidade de obter informação relevante para a investigação e de aprofundar especificidades da funcionalidade psíquica, familiar, social e comunitária do participante face ao comportamento alimentar e colmatar algumas das insuficiências, caraterísticas de instrumentos estruturados como são os questionários fechados.

\section{Questionário Sociodemográfico}

Questões orientadas para recolha de informação sobre categorias como idade, 
escolaridade, condição familiar, peso, estatura.

\section{Questionário do Comportamento Alimentar (QCA)}

Questionário constituído por 33 afirmações sobre comportamento alimentar, cuja resposta consistia em assinalar com "Sim" ou "Não" estar de acordo com a respetiva afirmação. Questionário também muitas vezes designado de "Questionário Holandês do Comportamento Alimentar" ou "Dutch Eating Behavior Questionnaire (DEBQ)" criado, em $1986^{56}$ e $2008^{57}$, com a finalidade de abordar os comportamentos de restrição da ingestão alimentar, ingestão externa e ingestão emocional. Mais tarde V. Viana e S. Sinde, $(2003)^{58,59}$ traduziram e validaram o "Questionário Holandês do Comportamento Alimentar (D.E.B.Q.)" para a população portuguesa. Versão que avalia 3 dimensões: Restrição Alimentar 10 afirmações; Ingestão Externa - 10 afirmações e Ingestão Emocional - 13 afirmações. A dimensão "Restrição Alimentar" está orientada para detectar atitudes e comportamentos assumidos pelo respondente que visam conscientemente restringir a quantidade de comida ingerida às refeições. A dimensão "Ingestão Externa" dirige-se à detecção de estímulos e situações externos que ativam a ingestão de alimentos, incluindo a ingestão de quantidades superiores ao necessário. A dimensão "Ingestão Emocional" visa detectar comportamentos de ingestão alimentar motivados por determinados estados emocionais, predominantemente por emoções e sentimentos de sinal negativo; é como uma forma de compensação em que a ingestão alimentar faz o sujeito sentir-se melhor, ou pelo menos reduzir a emoção ou sentimento negativo que está a experimentar naquele momento ou período temporal.

Nessa versão do questionário os autores que o validaram para a população portuguesa usaram a escala tipo Likert de 5 pontos (1 - "Nunca"; 5 - "Muito
Frequente"). Nessa investigação concreta manteve-se praticamente a mesma estrutura semântica das questões, tendo sido feitos alguns ajustes com o intuito de simplificar a compreensão dos respondentes, principalmente de crianças do $1^{\mathrm{o}}$ ciclo em que o domínio da língua portuguesa escrita ainda revela certas dificuldades.

Entretanto, na versão utilizada do QCA, após o respondente ter respondido com "Sim" ou "Não" à respectiva afirmação, solicita-se que atribua um valor de intensidade ou frequência que corresponda à força com que esse tipo de indutor exerce no seu comportamento face à ingestão alimentar. A escala de intensidade ou frequência implementada foi de 0 a 5 pontos, em que 0 corresponde à ausência de intensidade ou nunca, o 1 à existência de intensidade fraca ou poucas vezes, o 2 à intensidade ligeira, maior que fraca e menor que a média ou algumas vezes, o 3 corresponde à intensidade média ou várias vezes, o 4 à intensidade bastante significativa ou frequente $\mathrm{e}$ o 5 à intensidade forte ou muito frequente.

\section{Resultados}

$\mathrm{Na}$ tabela 2, em seguida, inscrevem-se os valores obtidos pelo grupo de sobrepeso / obesidade infantil e pelo grupo de controle. Os valores inscritos reportam-se ao índice de massa corporal (IMC) e às três dimensões do questionário de comportamento alimentar (QCA): restrição alimentar, ingestão externa e ingestão emocional. Em cada uma dessas três dimensões são reportados quatro indicadores: a quantidade de "sim" e a quantidade de "não" que os participantes de ambos os grupos assinalaram; a intensidade integral, que é a soma das intensidades assinaladas na totalidade das afirmações da respectiva dimensão; a intensidade média, que é a média obtida, resultante da divisão da soma integral de intensidades pela quantidade de questões constituintes da respectiva dimensão, assinaladas com resposta "sim". 
Tabela 2. Determinantes do comportamento alimentar em crianças e adolescentes com sobrepeso - obesidade infantil e do grupo de controle.

\begin{tabular}{|c|c|c|c|c|c|c|c|}
\hline \multicolumn{8}{|l|}{ Grupos } \\
\hline \multirow{2}{*}{ Categorias } & \multicolumn{2}{|c|}{$\begin{array}{c}\text { Sobrepeso / } \\
\text { Obesidade infantil }\end{array}$} & \multicolumn{2}{|c|}{$\begin{array}{l}\text { Grupo de } \\
\text { Controle }\end{array}$} & \multirow{2}{*}{ 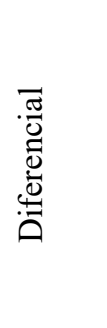 } & \multirow{2}{*}{ 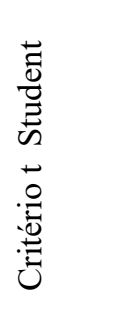 } & \multirow{2}{*}{ 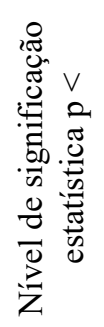 } \\
\hline & $\mathrm{M}$ & DP & $\mathrm{M}$ & DP & & & \\
\hline $\begin{array}{l}\text { Índice de Massa Corporal } \\
\text { (IMC) }\end{array}$ & 29,21 & 3,87 & 19,60 & 2,93 & 9,60 & 14,95 & 0,000 \\
\hline $\begin{array}{l}\text { Restrição alimentar; } \\
\text { quantidade "Sim" }\end{array}$ & 2,82 & 3,23 & 3,08 & 3,00 & $-0,46$ & $-0,72$ & 0,472 \\
\hline $\begin{array}{l}\text { Restrição alimentar; } \\
\text { quantidade "Não" }\end{array}$ & 7,18 & 3,37 & 6,92 & 3,00 & 0,26 & 0,39 & 0,693 \\
\hline $\begin{array}{l}\text { Restrição alimentar; } \\
\text { Intensidade Integral }\end{array}$ & 5,70 & 8,39 & 7,84 & 8,58 & $-2,14$ & $-1,26$ & 0,211 \\
\hline $\begin{array}{l}\text { Restrição alimentar; } \\
\text { Intensidade Média }\end{array}$ & 1,13 & 1,15 & 2,21 & 1,61 & $-1,08$ & $-3,99$ & 0,000 \\
\hline $\begin{array}{l}\text { Ingestão alimentar externa; } \\
\text { quant. "Sim" }\end{array}$ & 7,20 & 2,85 & 5,04 & 2,27 & 2,16 & 3,99 & 0,000 \\
\hline $\begin{array}{l}\text { Ingestão alimentar externa; } \\
\text { quant. "Não" }\end{array}$ & 2,80 & 2,85 & 4,96 & 2,27 & $-2,16$ & $-3,99$ & 0,000 \\
\hline $\begin{array}{l}\text { Ingestão alimentar externa; } \\
\text { Int. Integral }\end{array}$ & 25,86 & 15,95 & 16,24 & 12,00 & 9,62 & 3,40 & 0,001 \\
\hline $\begin{array}{l}\text { Ingestão alimentar externa; } \\
\text { Intens. Média }\end{array}$ & 3,19 & 1,26 & 2,97 & 1,14 & 0,22 & 0,96 & 0,341 \\
\hline $\begin{array}{l}\text { Ingestão alimentar emocional; } \\
\text { quant. "Sim" }\end{array}$ & 4,58 & 3,80 & 3,68 & 3,71 & 0,90 & 1,08 & 0,284 \\
\hline $\begin{array}{l}\text { Ingestão alimentar emocional; } \\
\text { quant. "Não" }\end{array}$ & 8,42 & 3,91 & 9,32 & 3,71 & $-0,90$ & $-1,14$ & 0,259 \\
\hline $\begin{array}{l}\text { Ingestão alimentar emocional; } \\
\text { Int. Integral }\end{array}$ & 14,44 & 14,58 & 8,54 & 10,89 & 5,90 & 2,25 & 0,029 \\
\hline $\begin{array}{l}\text { Ingestão alimentar emocional; } \\
\text { Int. Média }\end{array}$ & 2,50 & 1,48 & 2,15 & 1,59 & 0,34 & 1,07 & 0,287 \\
\hline
\end{tabular}

Dos resultados inscritos na tabela 2 verifica-se que o índice de massa corporal (IMC) entre o grupo de participantes com diagnóstico de sobrepeso ou obesidade infantil e o grupo de controle, constituído por participantes, os quais são referência da norma, apresenta diferenças estatisticamente significativas $(\mathrm{p}<0.000)$, sendo os valores bastante mais expressivos quando os participantes têm sobrepeso ou obesidade infantil. É importante especificar que de acordo com os referenciais do IMC no grupo de sobrepeso / obesidade cerca de $50 \%$ dos participantes enquadram-se no diagnóstico de sobrepeso e outros $50 \%$ no diagnóstico de obesidade, se atentarmos que $18 \%$ apresentam IMC entre 29 e 30; daí pode-se incorporar na obesidade, juntando aos $32 \%$ com valores claramente acima de 30 .

Na dimensão "Restrição Alimentar" do questionário de comportamento alimentar (QCA) a média de respostas "sim" é aproximadamente de $3 / 10$, ou seja, apenas consideraram estar de acordo, entre as 10 afirmações, que poucas são as que os levam a fazer algo de específico para restringir a quantidade de alimentos ingeridos, havendo semelhança expressiva em ambos os grupos e, por isso, também não se revela diferença estatisticamente significativa $(\mathrm{p}<0.472)$. Em contrapartida, a quantidade de "não" às afirmações relacionadas com comportamentos de 
restrição alimentar é de 7/10, em ambos os grupos, verificando-se a inexistência de diferenças estatisticamente significativas ( $p$ $<0.693$ ).

A intensidade integral da dimensão "Restrição Alimentar", a qual consiste no somatório das intensidades atribuídas pelos participantes às afirmações respondidas com "sim", não é muito diferente em ambos os grupos e, por isso, também não se verificam diferenças estatisticamente significativas ( $p<0.211)$. Contudo, a intensidade média, que é a média das médias das intensidades obtidas pelos participantes nas afirmações respondidas com "sim", já apresenta diferença estatisticamente significativa entre o grupo de participantes com sobrepeso / obesidade e o grupo de controle $(p<0.000)$. A ausência de diferença estatisticamente significativa na intensidade integral e existência na intensidade média explica-se pela elevada dispersão de valores relativa à intensidade integral, sendo a dispersão atenuada quando se transforma uma soma integral de intensidades numa média dessas intensidades no mesmo participante.

Embora haja semelhança nos valores obtidos por ambos os grupos da investigação denota-se ligeiramente maior expressividade no grupo de controle quanto à dimensão "Restrição Alimentar"; isso explica haver maior, ainda que ligeira, preocupação e cuidados a ter no grupo de controle, no que concerne a evitar em certas situações a ingestão alimentar.

$\mathrm{Na}$ dimensão "Ingestão Alimentar Externa" a média de respostas "sim" é de 7/10 e "não" 3/10 das afirmações no grupo de participantes com sobrepeso ou obesidade e de 5/10 para o "sim" e para o "não" no grupo de controle, existindo diferença estatisticamente significativa ( $p$ $<0.000$ ) em ambos os casos.

A intensidade integral da dimensão "Ingestão Alimentar Externa" é mais expressiva no grupo de participantes com sobrepeso / obesidade, sendo relativamente ao grupo de controle a diferença estatisticamente significativa $(p<0.001)$.
Entretanto, a intensidade média é sensivelmente idêntica em ambos os grupos da investigação, não sendo a diferença estatisticamente significativa $(\mathrm{p}<0.341)$. Nesse caso a média das intensidades por participante parece uniformizar o valor, tornando-o muito próximo em ambos os grupos e, por conseguinte, não significativa a diferença. Já a intensidade integral, havendo mais questões respondidas com "sim" no grupo dos participantes com sobrepeso, mesmo que a intensidade atribuída a cada afirmação seja igual em ambos os grupos, a diferença acaba por ser significativa.

$\mathrm{Na}$ dimensão "Ingestão Alimentar Emocional" a média de respostas "sim" é de 4,5/13 e "não" é 8,5/13 no grupo de participantes com excesso de peso e "sim" 3,6/13 e "não" 9,4/13 no grupo de controle, inexistindo diferenciação estatisticamente significativa ( $<$ <.284) para o "sim" e ( $p$ $<0.259$ ) para o "não".

A intensidade integral da dimensão "Ingestão Alimentar Emocional" é mais expressiva no grupo de participantes com sobrepeso / obesidade, quase o dobro, relativamente ao grupo de controle, sendo a diferença estatisticamente significativa ( $p$ $<0.029$ ). Entretanto, a intensidade média, embora mais expressiva no grupo com excesso de peso, a diferença é pouca relativamente ao grupo de controle; por isso, não é estatisticamente significativa ( $p$ $<0.287$ ). A explicação para esse fenômeno de diferenciação estatística entre a intensidade integral e a intensidade média é a mesma adotada na dimensão "Ingestão Alimentar Externa".

\section{Discussão}

Nesta investigação o índice de massa corporal (IMC) apresenta-se claramente como um referencial de diagnóstico importante; primeiro na diferenciação de crianças e adolescentes com sobrepeso e com obesidade e, em segundo lugar, como indicador da possível existência de inadequações do comportamento alimentar. $\mathrm{O}$ recurso às 
informações recolhidas no âmbito da entrevista e observação clínico-psicológica inferem que todos os participantes do grupo com sobrepeso / obesidade tinham comportamento alimentar desadequado da sua condição etária, estilo e rotina alimentar diária, tendo em conta as suas necessidades nutricionais e a realidade da sua vida. São frequentes as queixas dos pais sobre comportamentos dos filhos como comer às escondidas, repetição, comendo em demasia na hora da refeição, ao longo do dia devoram pacotes de bolachas, preferências por alimentos ricos em gorduras e hidratos de carbono; chegando a amuar e até ofender quem se recusa a permitir que leve avante as suas intenções alimentares, dizendo, por vezes com raiva e ódio, "queres é que eu passe fome, matar-me à fome". Muitas vezes a discórdia alimentar entre pais e filhos é motivo para gerar tensão emocional nas relações interpessoais e na funcionalidade familiar. No entanto, há casos em que o conflito por causa do comportamento alimentar dos filhos não existe, porque os pais consideram que é normal, que eles também eram assim e estão bem; que o querer controlar mais os alimentos e a quantidade ingerida é excesso de zelo dos profissionais de saúde.

De acordo com as investigações de vários autores, tendo em conta as teorias do comportamento alimentar, o índice de massa corporal se correlaciona positivamente com a dimensão "Restrição Alimentar" ${ }^{\prime \prime}$. Significa que quando há um IMC mais elevado a tendência é para existirem comportamentos orientados para a restrição alimentar; provavelmente, por se tomar consciência em determinada fase da vida que tem cometido erros e exageros do foro alimentar e que deverá adotar medidas para corrigir os seus hábitos e estilo alimentar.

A inexistência de diferença estatisticamente significativa na dimensão "Restrição Alimentar", indicia que, em termos qualitativos, tanto nos participantes do grupo de sobrepeso e obesidade como nos do grupo de controle, a predisposição psicológica, as atitudes e os comportamentos orientados à restrição alimentar, ou seja, para evitar ou reduzir a quantidade de alimentos ingeridos, são escassos, salvo em uma ou outra situação. A própria frequência ou intensidade integral atribuída pelos participantes às afirmações respondidas com "sim", não é diferente em ambos os grupos, não se verificando diferenças estatisticamente significativas.

Existe semelhança quer na expressão qualitativa, quer na quantitativa entre valores obtidos por ambos os grupos da investigação, embora se note expressividade ligeiramente maior no grupo de controle. Isso explica que as crianças e adolescentes do grupo de controle evidenciam, ainda que ligeira, maior preocupação e cuidados no que concerne a evitar,, em certas situações, a ingestão alimentar.

A consciência e respectiva percepção adequada de estar em transgressão relativamente ao seu comportamento alimentar face a diversas situações e circunstâncias da vida saudável são exigidas aos sujeitos cuja estrutura orgânica já se encontra na condição de sobrepeso ou de obesidade e não propriamente àqueles em que essa condição não existe. Naturalmente, as crianças e adolescentes cujo comportamento alimentar é adequado à manutenção do estilo de vida saudável, e que não apresentam qualquer indicador negativo relacionado com $\mathrm{o}$ excesso de peso ou obesidade, não necessitam fazer qualquer esforço volitivo para restringir a ingestão de comida, ter preocupações específicas, nem promover a mudança de atitudes e comportamentos face à atividade alimentar.

Por conseguinte, os resultados obtidos pelas crianças e adolescentes com sobrepeso ou obesidade na dimensão "Restrição Alimentar" indiciam a existência de déficit de conscientização adequada e provavelmente de percepção distorcida do seu comportamento alimentar; processos que promovem a possibilidade de formação de representações sociopsicológicas e 
atitudes incongruentes e dissonantes com as situações de ingestão alimentar, concernentes ao estilo de vida saudável. O reforço e cristalização de atitudes e representações sociopsicológicas do comportamento alimentar, incongruentes com estilos de vida saudáveis, são terreno propício à instalação de predisposições psíquicas desadequadas que fomentam a continuidade de condutas alimentares transgressoras face à real necessidade dos sujeitos que delas são dependentes. Debilidade ou ausência de condições psicológicas para efetivar o controle e a restrição alimentar requeridos pelos indicadores de vida saudável corroborados pelos resultados obtidos na dimensão "Ingestão Alimentar Externa".

As diferenças estatisticamente significativas apuradas na dimensão "Ingestão Alimentar Externa", nas afirmações respondidas com "sim" e com "não", entre os grupos de participantes envolvidos na investigação, indiciam que as crianças e adolescentes diagnosticadas com sobrepeso ou obesidade têm propensão para comportamentos de ingestão alimentar desencadeados por estímulos externos relacionados com propriedades sensoriais de natureza psicofísica dos produtos, mas também por situações de natureza psicocultural que atualizam os desejos ou necessidades na estrutura funcional interna do sujeito da ação como sistema bio-neurofisio-psico-sociocultural ${ }^{2,4,61}$.

A diferença estatisticamente significativa na intensidade integral da dimensão "Ingestão Alimentar Externa" com maior pendor expressivo no grupo de participantes com sobrepeso / obesidade, demonstra a força impulsionadora das imagens e representações psicológicas exercida sobre o comportamento alimentar do sujeito da ação despertada e atualizada pelo poder atrativo das propriedades sensoriais dos produtos e pelo significado pessoal e valor psicossociocultural adquirido. Fenômenos e mecanismos de certo modo suportados cientificamente pelas investigações desenvolvidas no âmbito da regulação do comportamento alimentar com base nas caraterísticas, processos e funções do apetite e da saciedade como entidades sistémicas ${ }^{48,49}$.

A intensidade média apurada nessa dimensão não obteve diferenciação estatística significativa porque reduz a determinação do valor num intervalo muito pequeno, não sendo assim a diferença numérica suficientemente expressiva; também se poderá explicar com base nos mecanismos de defesa do seu "ego" numa situação de avaliação, procurando não reconhecer a verdadeira força que $o$ estímulo tem em si, selecionando valores baixos, os quais acabam por coincidir com aqueles atribuídos pelos participantes do grupo de controle.

$\mathrm{Na}$ dimensão "Ingestão Alimentar Emocional" as respostas "sim" e "não" às afirmações, por ambos os grupos da investigação, não alcançaram diferenças estatisticamente significativas. A explicação prende-se com o fato de esta dimensão ser constituída por afirmações cujos indutores do comportamento alimentar são estados emocionais específicos (tédio, irritação, depressão, raiva, e outros) e além de serem apenas algumas pessoas em que a ingestão alimentar descontrolada é desencadeada por estarem a vivenciar um estado emocional específico, e a tipologia do estado emocional também varia de pessoa para pessoa. Nessas condições, a quantidade de afirmações é reduzida, resumindo-se a não mais de 4 participantes, sendo na maior parte deles reduzida a zero; porque nesses a indução mais provável relaciona-se com fatores de ingestão alimentar externa.

Consequentemente, devido à elevada variabilidade de escolha do estado emocional indutor, e até ausência, a dispersão também é bastante elevada e, por isso mesmo, a existência de valor diferencial não é suficiente para que surja a diferença estatisticamente significativa. Entretanto, a intensidade integral na dimensão "Ingestão Alimentar Emocional" é mais expressiva no grupo de participantes 
com sobrepeso / obesidade, quase o dobro, relativamente ao grupo de controle, sendo a diferença estatisticamente significativa, mas na intensidade média não é.

A análise qualitativa da atribuição de intensidade à força que o respectivo indutor exerce no sujeito da ação e os comentários que tece acerca da condição estimuladora, revela que nos participantes do grupo de sobrepeso / obesidade, em alguns deles, o estado emocional vivido em determinados momentos desencadeia comportamentos alimentares desadequados caraterizados por tendência voraz de ingestão alimentar, sendo que essa tipologia de indução praticamente não tem expressão no grupo de controle, apenas alguns reportam que em algumas situações de estresse, saturação ou tédio podem recorrer à ingestão de certos alimentos, como chocolate e outros doces, instrumentalmente em situações muito específicas; por isso acabam por atribuir algum valor de intensidade, mas não sendo prática corrente e não deixando vestígios que indiciem repetição recorrente.

Apesar de não existir diferenças estatisticamente significativas entre os grupos desta investigação, quanto à dimensão "ingestão alimentar emocional" o comportamento alimentar desadequado, face às situações envolventes, induzido e desencadeado por estados emocionais específicos, está presente em vários participantes do grupo com sobrepeso / obesidade; fenómeno confirmado por outros autores que mostraram haver pessoas em que a vivência interior de certos estados emocionais, habitualmente de conotação negativa, como estresse, tédio, saturação, irritação, depressão, indignação, raiva, fúria, ódio e outros, induzem comportamentos alimentares desadequados, além de outros tipos de comportamentos inapropriados face às situações e circunstâncias envolventes $^{39,40,41}$. No entanto, também a obesidade, como objeto da percepção dos próprios sujeitos portadores, pode desencadear problemas de saúde mental, cujas manifestações são frequentemente ansiedade e depressão, resultando, quase sempre da insatisfação corporal, da autoimagem negativa, da baixa autoestima ${ }^{20,21,26}$. Portanto, a obesidade infantil e adulta é considerada um problema de saúde pública ${ }^{10,13}$, mas também social, devido ao estigma atribuído às pessoas com excesso de peso ${ }^{16}$.

Para compreender a obesidade infantil e pensar intervenções eficazes devese considerar todos os elementos que envolvem o fenômeno e focar a atenção na pessoa em vez da obesidade ${ }^{11}$.

A obesidade infantil acarreta prejuízos para a saúde, sendo que os distúrbios provocados pelo excesso de peso comprometem significativamente a qualidade de vida futura ${ }^{33}$.

O sucesso das estratégias de intervenção no sobrepeso/ obesidade depende particularmente dos conhecimentos acerca dos determinantes comportamentais e psicossociais dos sujeitos a quem se dirige a ação ${ }^{34}$.

$\mathrm{O}$ ato de comer é um processo biológico, mas o que determina a forma como os seres humanos se comportam e fazem as suas escolhas alimentares é a cultura $^{36,37}$. Os aspectos determinantes do comportamento alimentar não se assentam exclusivamente em mecanismos endógenos de natureza biológica; daí a importância da compreensão da experiência subjetiva do apetite e as preferências por alimentos específicos. A regulação do apetite está associada ao estado de saciedade do sujeito, havendo participação tanto de mecanismos endógenos de natureza biológica como de natureza psicológica e também de mecanismos exógenos de natureza social, cultural, ambienta ${ }^{48}$. Por conseguinte, os aspectos determinantes do comportamento alimentar poderão encontrar-se a vários níveis de funcionalidade do sistema bioneuro-fisio-psico-socio-cultural, reportarse a várias dimensões da atividade interior, envolvendo várias esferas e componentes estruturais ${ }^{4}$ 
$\mathrm{Na}$ tentativa de conceitualização teórica sistêmico-integrativa da funcionalidade psíquica do comportamento alimentar, na condição de sobrepeso ou obesidade, apenas será reportado o nível psicológico, a dimensão da atividade psíquica e espaços também relativos à consciência, às esferas cognitiva, emocional, motivacional e volitiva, os componentes da estrutura funcional da atividade psíquica envolvida na entidade nosológica "sobrepeso" ou "obesidade" e quando possível também os vetores controlo-regulativo e reflexivo-adaptativo ${ }^{4}$.

$\mathrm{Na}$ atividade funcional do sujeito ao nível psíquico, a qual se encontra envolvida no processo de formação, desenvolvimento, consolidação e manutenção das entidades "sobrepeso" e "obesidade" como «dependência», papel relevante assumem as esferas cognitiva, emocional, motivacional e volitiva, as quais no início gozam de total autonomia funcional relativamente à "dependência", mas à medida que o comportamento alimentar se torna desadequado progressivamente assiste-se à imersão de emoções, à colonização do funcionamento cognitivo, à submissão de motivações, potenciando mais e mais a motivacionalidade inapropriada, à subsidiação dos processos volitivos de modos que a ação destes torna-se refém da dependência, contribuindo para subsidiar o seu desenvolvimento no sentido de se tornar uma estrutura funcional da atividade psíquica do sujeito mais e mais instalada, ganhando consistência e domínio da vida pessoal e até social dos sujeitos portadores da condição "obesidade". Nesse processo, papel relevante é desempenhado pela dimensão "consciência", a qual avalia e confere maior ou menor aceitação da condição. Além disso, no plano sistémicointegrativo da dimensão da personalidade / individualidade, envolvendo a participação articulatória "consciência", é também importante a ação vetorial desempenhada pelas funções integrais de reflexividade, controlabilidade, regulatividade e adaptabilidade do sistema bio-neuro-psicosocio-cultural ${ }^{4}$.

Figura 1. Concepção sistêmico-integrativa do comportamento alimentar na obesidade.

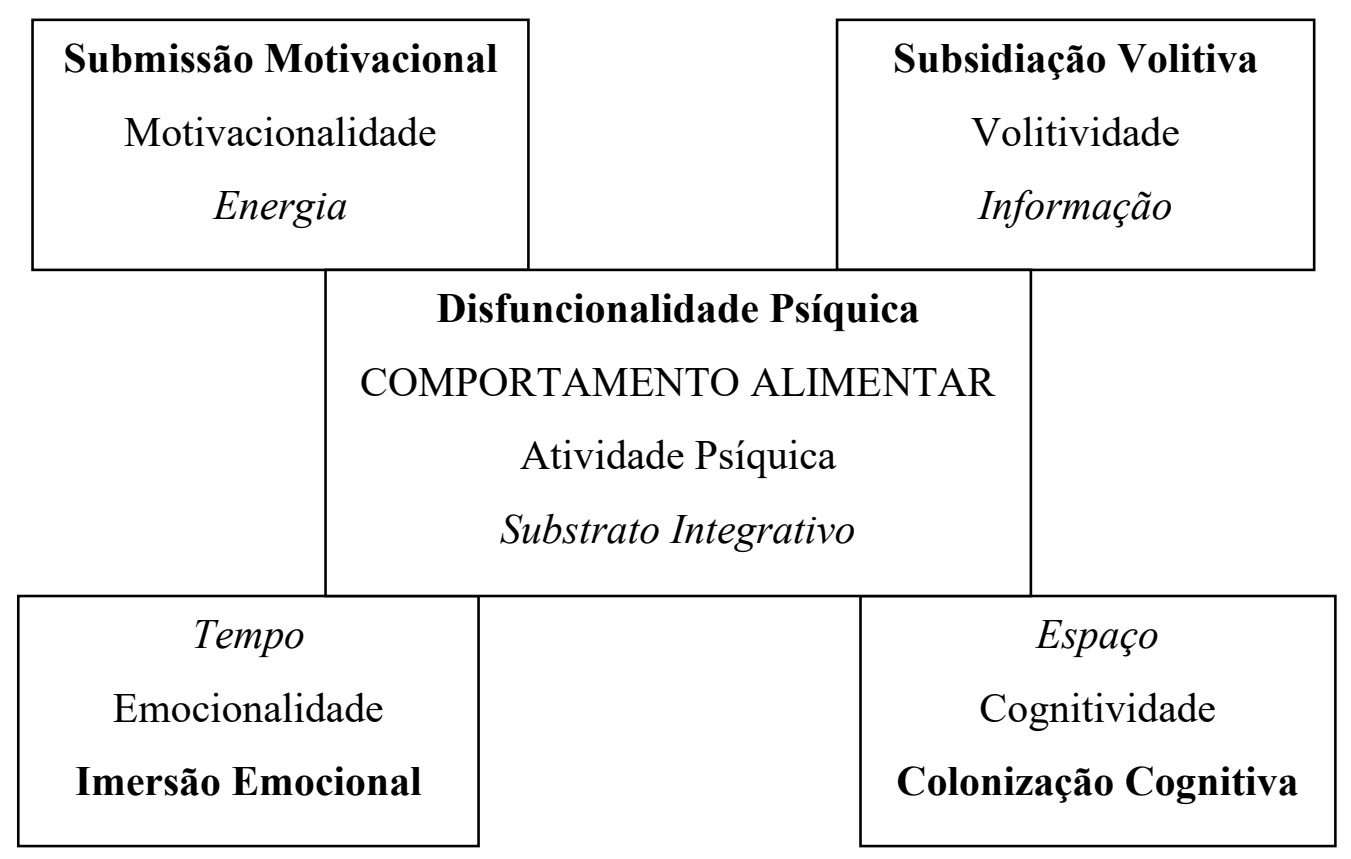

Conforme os componentes emocional, cognitivo, motivacional e volitivo da atividade psíquica apresentam sinais de disfuncionalidade, ao nível 
psíquico, psicossocial, sociocultural, comportamental, envolvida nas mudanças do comportamento alimentar que o tornam desadequado, avança-se no território da dependência, em termos psicosociocomportamentais, revelando-se externamente no produto que é o sobrepeso e a obesidade.

Nesta investigação o IMC indicia existência de sobrepeso ou obesidade decorrentes, em grande parte, do comportamento alimentar desadequado, o qual pode estar ancorado em atividade psíquica e atitudes disfuncionais refletidas no modo como os sujeitos controlam e regulam as suas ações de restrição alimentar, determinada pela tomada de consciência de cometer exageros, e ingestão alimentar determinada por fatores de ordem sensorial e emocional empolgados que atualizam a sua força com base na percepção direta de certos alimentos e suas propriedades ou de representações ou memórias, formadas, desenvolvidas e consolidadas por meio de hábitos, usos e costumes e tradições inerentes à sua condição sociocultural.

Por conseguinte, os resultados da investigação poderão orientar na elaboração de ações psicológicas promotoras da correção do comportamento alimentar e também de estratégias educacionais preventivas.

\section{Conclusão}

Os participantes da investigação com sobrepeso / obesidade tinham comportamento alimentar desadequado da sua condição etária, necessidades nutricionais e estilo de vida.

O comportamento de restrição alimentar idêntico entre os participantes do grupo de sobrepeso/obesidade e o grupo de controle infere ausência de predisposições psicológicas e atitudes para evitar ou reduzir a quantidade de alimentos ingeridos.
Por conseguinte, os valores obtidos pelas crianças e adolescentes com sobrepeso ou obesidade na dimensão "Restrição Alimentar" decorrem do déficit de consciencialização adequada e provavelmente da percepção distorcida do seu comportamento alimentar.

As crianças e adolescentes com sobrepeso ou obesidade têm propensão para comportamentos de ingestão alimentar desencadeados por estímulos externos relacionados com propriedades sensoriais de natureza psicofísica dos produtos, mas também por situações de natureza psicocultural que atualizam os desejos ou necessidades na estrutura funcional interna do sujeito da ação como sistema bio-neurofisio-psico-sociocultural.

Os indutores emocionais do comportamento alimentar diferem de pessoa para pessoa e apenas têm ação efetiva em algumas dessas pessoas; por isso, elevada variabilidade do estado emocional indutor e dispersão avultada explicam ausência de diferença estatisticamente significativa. Contudo, no grupo de sobrepeso / obesidade, há participantes em que o estado emocional vivido em determinados momentos desencadeia comportamentos desadequados orientados para ingestão alimentar desnecessária.

O comportamento alimentar desadequado e conducente ao sobrepeso ou obesidade, à luz da concepção sistêmicointegrativa da funcionalidade psíquica, decorre de mudanças progressivas operadas no interior do sistema que envolvem processos de imersão emocional, colonização cognitiva, submissão motivacional e subsidiação volitiva, podendo com o tempo de estágio e habituação vir a estabelecer-se na condição de dependência e alterar a ação dos vetores controlo-regulativo e reflexivo-adaptativo. 


\section{Referências}

1. GanzenVA. Descrições sistémicas em psicologia. Leninegrado: Universidade Estatal de Leninegrado; 1984.

2. LomovBF. Problemas teóricos e metodológicos da psicologia. Moscovo: Ciência; 1984.

3. Morin E. La complexité humaine. Paris: Flammarion; 1994.

4. Pereira FO. Teoria Sistémico-Integrativa do Psiquismo Humano. Revista Teoría y Crítica de la Psicología. 2018; n. 10, 1-23. http://www.teocripsi.com/ojs/(ISSN:2116-3480).

5. Ahrebs W, Pigeot I. Fatores de risco da obesidade infantil: Lições do Estudo Europeu IDEFICS. In ML Frelut (Ed.), The ECOG`seBookonChildandAdolescentObesity;2015. Retirado de ebook.ecog-obesity.eu

6. Médicos do Mundo. Como se previne a obesidade infantil? $\mathrm{s}$. $\mathrm{d}$. Médicosdomundo.pt/noticia/como-se-previne-obesidade-infantil. Recuperado 3 de Julho de 2020.

7. SNS (Serviço Nacional de Saúde). Obesidade Infantil em Portugal, 2019. sns.gov.pt/noticias/2019/07/10/Portugal-obesidade-infantil-2/. https:/www.dgs.pt/. Recuperado em 3 de julho de 2020.

8. ABESO (Associação Brasileira para o Estudo da Obesidade e da Síndrome Metabólica). Mapa da obesidade. Abeso, 2015. Disponível em: http://www.abeso.org.br/atitudesaudavel/mapa-obesidade.

9. Moraes JM, Caregnato RCA, Schneider DS. Qualidade de vida antes e após a cirurgia bariátrica. Acta Paulista de Enfermagem. 2014;27, 157-164. doi: 10.1590/19820194201400028.

10. Frontzek LGM (Org). Obesidades e psicologia. Porto Alegre: Juruá; 2015.

11. Frontzek LGM, Bernardes LR, Modena CM. Obesidade infantil: Compreender para melhor intervir. Rev. Abordagem Gestaltica. [online]. 2017; vol.23, n.2, pp. 167-174. ISSN 18096867.

12. Nunes MA, Appolinario JC, Abuchaim ALG, Coutinho W (eds). Transtornos alimentares e obesidade. Porto Alegre: Artmed; 2006.

13. Freitas FC. A janela da escuta. Relato de experiência clínica. Belo Horizonte: Editora Scriptum; 2014.

14. Melo ED, Luft VC, Meyer F. Obesidade Infantil: como podemos ser eficazes. Jornal de Pediatria. Porto Alegre - Rio Grande do Sul. 2003; v. 80, n. 3, pag: 1 a 16, maio/junho.

15. Reis CEG, Vasconcelos IAL, Barros JDN. Políticas públicas de nutrição para o controle da obesidade infantil. Revista Paulista de Pediatria. 2011; 29, 625-33. doi: 10.1590/S010305822011000400024.

16. PoulainJP. Sociologia da obesidade. São Paulo: Editora Senac; 2013.

17. Carvalho EADA, Simão MTJ, Fonseca MC, Andrade RGD, Ferreira MSG, Silva AF, Fernandes BS. Obesidade: aspectos epidemiológicos e prevenção. Revista Médica de Minas Gerais. 2013; 23,74-82. doi: 10.5935/2238-3182.20130012.

18. Marchi-Alves LM, Yagui CM, Rodrigues CS, Mazzo A, Rangel EML, Girão FB. Obesidade infantil ontem e hoje: importância da avaliação antropométrica pelo enfermeiro. Escola Anna Nery 2011; 15, 238-244. doi: 10.1590/S1414-81452011000200004.

19. Melo TR., Jansen AK., Pinto R. DMC., Morales R. R. D., Morales NM, Prado MM, Silva CHMD. Qualidade de vida de cuidadores de crianças e adolescentes com sobrepeso ou obesidade. Revista Escola de Enfermagem USP. 2011; 45, 319-26. doi: 10.1590/S008062342011000200003.

20. Andrade TDM, Moraes DEBD, Ancona-Lopez F. Problemas Psicológicos e Psicodinâmicos de crianças e adolescentes obesos: relato de pesquisa. Psicologia: Ciência e Profissão. 2014; 34, 126-141. doi: 10.1590/S1414-98932014000100010. 
21. Turco G, Bobbio T, Reimão R, Rossini S, Pereira H, Barros Filho A. Quality of life and sleep in obese adolescents. Arquivos de neuro-psiquiatria. 2013; 71, 78-82. doi: 10.1590/S0004-282X2013005000008

22. Assunção MCF, Muniz LC, Schäfer AA, Meller FDO, Carús JP, Quadros LDCMD, Menezes AMB. Tornar-se obeso na adolescência pode trazer consequências à saúde mental? Caderno de Saúde Pública. 2013; 29, 1859-1866. DOI: 10.1590/0102$311 \mathrm{X} 00135812$.

23. Crivelaro SHR, Tuma MÂF. Nível de atividade física, obesidade e bullying em escolares. Corpo e Movimento Educação Física. 2015; 6, 29-35.

24. Scutti CS, Seo GY, Amadeu RS, SampaioRF. O enfrentamento do adolescente obeso: a insatisfação com a imagem corporal e o bullying. Revista da Faculdade de Ciências Médicas de Sorocaba. 2014;16, 130-133.

25. Borges F, Barreto MS, Reis P, Viera CS, Marcon SS. Percepções e atitudes de crianças que vivenciam a obesidade. Revista Rene. 2018; 19. DOI: 10.15253/2175-6783.2018193261 www.revistarene.ufc.br

26. Melca IA, Fortes S. Obesidade e transtornos mentais: construindo um cuidado efetivo. Revista Hospital Universitário Pedro Ernesto. 2014; 13, 18-25. doi: 10.12957/rhupe.2014.9794

27. Bellodi AC. Obesidade em crianças e adolescentes: temperamento, estresse, coping e risco psicossocial familiar (Tese de Doutorato em Psicologia). Campinas, SP: Pontifícia Universidade Católica de Campinas; 2018.

28. Nogueira TFD, Zambon MP. Reasons for non-adherence to obesity treatment in children and adolescents. Revista Paulista de Pediatria. 2013; 31, 338-343. doi: 10.1590/S010305822013000300010.

29. Rocha M, Pereira H, Maia R, Silva E, Morais N, Maia E. Aspectos psicossociais da obesidade na infância e adolescência.Psicologia, Saúde \& Doenças. 2017; 18(3), 712-723 ISSN - 2182-8407 Sociedade Portuguesa de Psicologia da Saúde - SPPS - www.sp-ps.pt DOI: http://dx.doi.org/10.15309/17psd1807

30. Frontzek LGM. Percepção dos pais sobre a obesidade dos filhos: revisão integrativa que apontou caminhos para a intervenção. Belo Horizonte; 2016.

31. Camargo APPM., Azevedo BA, Antonio MARM. A não percepção da obesidade pode ser um obstáculo no papel das mães de cuidar de seus filhos. Ciência e Saúde Coletiva. 2013;18(2): 323-333.

32. Coradini AO, Moré CLOO, Scherer AA. Obesidade, família e transgeracionalidade: uma revisão integrativa da literatura. Nova Perspectiva Sistêmica. 2017; n. 58, p. 17-37, agosto 2017.

33. Souza JC. Factores que contribuem para o desenvolvimento da obesidade infantil: revisão integrativa. Artigo científico apresentado a TCC II do Curso de Enfermagem da Universidade Católica do Salvador. Salvador Ba; 2019.

34. Vaz AI, Silva DII, Rego CIII, Viana VIV. Determinantes comportamentais em crianças e adolescentes com diagnóstico de obesidade. Alimentação Humana. 2010; Vol. 16, $\mathrm{n}^{\mathrm{o}} 2$, Revista da SPCNA.

35. Viana V, Guimarães MJ, Teixeira MC, Barbosa MC. Aquisição e desenvolvimento de atitudes face à saúde na infância e adolescência. Acta Pediátrica Portuguesa. 2003; 34:27786.

36. Kiess W, Marcus C, Wabitsch M.Obesity in ChildhoodandAdolescence. PediatrAdolesc Med. 2004; 9:124-36.

37. Viana V, Candeias L, Rego C, Silva D. Comportamento Alimentar em Crianças e Controlo Parental: Uma Revisão Bibliográfica. Alimentação Humana. 2009; 15(1):9-16. 
38. Savage JS, Fisher JO, Birch LL. Parental InfluenceonEatingBehavior: ConceptiontoAdolescence. J Law Med Ethics. 2007; 35(1):22-34.

39. Dixon JB, Dixon ME, O’Brien PE. Depression in AssociationwithSevereObesity. American Medical Association. 2003; 163:2058-65.

40. Moschonis G, Grammatikaki E, Manios Y. Perinatal predictors of overweight at infancy and preschool childhood: the GENESIS study. Int J. Obes. 2008; 32:39-47.

41. Luiz AM, Gorayeb R, Júnior RR, Domingos M. Depressão, ansiedade e competência social em crianças obesas. Estudos de Psicologia; 2005.

42. Simões D, Meneses RF. Autoconceito em crianças com e sem obesidade. Psicologia: Reflexão e Crítica. 2007; 20 (2), 246-251. Disponível em: http://www.scielo.br/pdf/prc/ v20n2/a10v20n2.pdf.

43. Ricciardelli LA, MacCabe MP. Children's Body Image Concerns and Eating Disturbance: A Review oftheLiterature. Clin Psychol Ver. 2001; 21(3):325-44.

44. Wardl J, Cooke L. The Impact of obesity on psychological well-being. Best Practices \& Research Clinical Endocrinology \& Metabolism. 2005; 19(3):421-40.

45. Latner JD, Stunkard A, Wilson GT. Stigmatized Students: Age, Sex, and Etnicity Effects in the Stigmatization of Obesity. Obes Res. 2005; 13(17):1226-31.

46. Galindo EM, Carvalho AM. Translation, adaptation and internal consistency evaluation of the Eating Behaviours and Body Image Test for female children. Rev Nutr. 2007; 20 (1):4754.

47. Decaluwé V, Braet $\mathrm{C}$. The cognitive behavioural model for eating disorders: A direct evaluation in children and adolescents with obesity. Eating Behaviors. 2005; 6:211-20.

48. Sinopoulou V, Harrold J, Halford J. Significado e análise da saciedade na infância. In ML Frelut (Ed.), The ECOG's eBook on Child and Adolescent Obesity; 2015. Retirado de ebook.ecog-obesity.eu

49. Blundell JE, Burley VJ, Cotton JR, Lawton CL. Dietary-Fat and the Control of EnergyIntake - Evaluating the Effects of Fat on Meal Size and Postmeal Satiety. American Journal of Clinical Nutrition. 1993; 57(5):S772-S8.

50. Santos RA, Maranhão ThLG, Batista HMT. Obesidade infantil e abordagens em psicologia: Uma revisão da literatura. Revista Multidisciplinar e de Psicologia. 2016; V. 10, N. 30, Supl. 1, Julho/2016. Edição electrónica em http://idonline.emnuvens.com.br/id

51. Rolland-Cachera MF, Cole T. Measurements and definition. In: Burniat,W., Lissau, I.\& Cole, T. (Eds). The obese and overweight child.Cambridge:University Press; 2002, pp. 327.

52. Dietz WH, Robinson TN. Use ofthebodymass index (BMI) as a measureofoverweight in childrenandadolescents. J. Pediatr.1998; 132:191-3.

53. Must A, Jacque PF, Dallal GE, Bajema CJ, Dietz WH. Longtermmorbidityandmortalityofoverweightadolescents - a follow-up ofthe Harvard groupstudyof 1922 to 1935. New Engl J Med. 1992; 327:1350-5.

54. Rolland-Cachera MF, Deheeger M, Avons P, Guilloud-Bataille M, Patois E, Sempé M. Tracking adiposity patterns from 1 month to adulthood. Ann Hum Biol.1998; 14:219-22.

55. Garrow J, Webster J. Quetelet index (W/H?) as a measure of fatness. International Journal of Obesity \& Related Metabolic Disorders. 1985; 9, 147-153.

56. Van Strien T, Frijters J, Bergers G, Defares P. The Dutch Eating Behavior Questionnaire (DEBQ) for assessment ofrestrained, emotional, and external eating behavior. International Journal of Eating Disorders. 1986; 5, 295-315.

57. Van Strien T, Oosterveld P. The children's DEBQ for assessment ofrestrained, emotional, and external eating in 7- to 12-year-old children. International Journal of Eating Disorders. 2008; 41 (1),72-81. 
58. Viana V, Sinde S. Estilo Alimentar: Adaptação e validação do Questionário Holandês do Comportamento Alimentar. Psicologia, Teoria, Investigação e Prática. 2003; 1, 59-71.

59. Viana V, Sinde S. O Questionário Holandês do Comportamento Alimentar (D.E.B.Q.). In M. Gonçalves, M. Simões, L. Almeida \& C. Machado (Eds.), Avaliação Psicológica. Instrumentos validados para a população portuguesa,Vol. 1, pp. 99-112. Coimbra: Quarteto; 2003.

60. Pirke K, Laessle R. Restrainedeating. In A. Stunkard, \& T. Wadden (Eds.), Obesity: Theory and therapy (2nd ed., pp. 151-162). New York: Raven Press; 1993.

61. Ananev BG. Sensações e necessidades. Psicologia e pedagogia: Apontamentos científicos. Vol. 11, No 244, pp. 67-74. Leninegrado: Universidade Estatal de Leninegrado;1957.

\section{Como citar este artigo:}

Pereira FO. Aspectos psicológicos determinantes do comportamento alimentar em crianças e adolescentes com sobrepeso ou obesidade. Rev. Aten. Saúde. 2021; 19(69): 279-297. 